apy described may ensure protection from infection of new T lymphocytes generated from the patient stem cells under thymic influence. Such cells could contribute to significant degrees of immune reconstitution of the individual.

H.F.SEWELL F. WALKER

Department of Pathology,

University of Aberdeen,

Foresterhill, Aberdeen AB9 $2 X D, U K$

1. Curran, J.W. et al. Science 229, 1352 (1985).

2. Mariman, E.C.M. Nature 318, 414 (1985).

3. McDougal, J.S. et al. J. Immun. 135, 3151 (1985).

4. Dalgleish, A.G. et al. Nature 312, 763 (1984).

5 Weiss, R.A. et al. Nature 316, 69 (1985).

. Robert-Guroff, M. et al. Nature 316, 72 (1985).

7. Cobbold, S.P. et al. Nature 312, 548 (1984).

Spire, B. et al. Lancet ii, 899 (1984).

9. Hoxie, J.A. et al. Science 220, 1400 (1985)

\section{Immunosuppressants in patients with AIDS}

SIR-An interesting rationale for the treatment of acquired immune deficiency syndrome (AIDS) with the immunosuppressant cyclosporin $\mathrm{A}$ is based on the view that AIDS is an autoimmune disease $^{1}$. Immunosuppressive drugs would be designed to reduce virus replications and autoimmune processes triggered by retroviral envelope proteins that are bound to T4 molecules. Klatzmann and Montagnier state, however, that there is still no firm basis for such a therapeutic approach.

To this point, we are able to present a contribution that is experimentally corroborated by studies on neopterin excretion: measurement of neopterin levels provides a sensitive tool able to quantify activation of T-lymphocytes in vivo. Elevated neopterin levels are observed in virtually all diseases where activation of $\mathrm{T}$ cells is apparent. In vitro, neopterin is released exclusively from macrophages in response to specific stimulation with $\gamma$ interferon (IFN- $\gamma$ ) secreted by activated T-cells ${ }^{2}$.

Studies of neopterin excretion have demonstrated an activated cellular immune system in patients with AIDS, with AIDS related complex (ARC) and in risk-group members ${ }^{3-5}$. The frequency of elevated neopterin levels in risk groups is significantly correlated to the known AIDS risk factors: receptive anal intercourse, parenteral drug abuse and clotting factor substitution therapy in haemophiliacs. This elevation is observed also in recipients of multiple blood transfusions, infant children and in LAV/HTLV-III seronegative, apparently healthy riskgroup members 5 . Although the activation of the cellular immune system seems to contrast with the clinical presentation of AIDS, the high serum levels of $\alpha_{1}$ thymosin and of acid-labile IFN- $\alpha$ in AIDS and ARC patients and elevated cytotoxic T-lymphocyte activity against allogeneic lymphoblasts in homosexual men are consistent with activation ${ }^{6}$. These data argue for the view that T-cell-activation plays a crucial role in the development of AIDS. We conclude that conditions linked with T-cell activation are prerequisite for the development of AIDS. Permanent or multiple stimulations of the cellular immune system might represent the often discussed cofactor for progressive syndrome.

According to this concept, and as suggested by Klatzmann and Montagnier and demonstrated in vitro ${ }^{7}$, the activation and proliferation of T4-cells will determine how much and how fast virus replication will ensue.

Consequently, T-cell activation should be strictly avoided particularly in LAV/ HTLV-III seropositive individuals. Application of immunostimulative drugs such as interleukin-2 (IL-2), will exacerbate the course of disease by accelerating virus spreading and T4-depletion; the failure of IL-2 treatment is well known ${ }^{1}$. Regimens preventing activation of T-cells and applications of immunosuppressive agents (such as cyclosporin $\mathrm{A}$ ) that inhibit secretion of IL- 2 from T-cells, should be tested with adequate caution.

Finally, the activation of $\mathrm{T}$-cells by treatment can be readily assessed by measurement of serum or urinary neopterin concentrations which rise with immunostimulatory therapy and fall with immunosuppression.

Financial support by the Jubiläumsfonds der Österreichischen Nationalbank, P2591, is gratefully acknowledged.

A. Hausen

M.P. DIERICH

D. FuCHS

P. Hengster

G. REIBNEGGER

T. SCHULZ

E.R. WERNER

H. WACHTER

Institute of Medical Chemistry

and Biochemistry,

Institute of Hygiene,

University of Innsbruck,

A-6020 Innsbruck, Austria

1. Klatzmann, D. \& Montagnier, L. Nature 319, 10 (1986).

2. Huber, C. et al. J. exp. Med. 160, 310 (1984).

3. Wachter, H. et al. Hoppe Seyler's Z. physiol. Chem. 364 1345 (1983)

Fuchs, D et at, Lancet ii, 1130 (1985)

Wachter, $\mathrm{H}$. et al. Lancet $\mathrm{i}, 97(1986)$

6. Tung, K.S.K. et al. J. Immun. 135, 3163 (1985)

7. McDougal, J.S. et al. J. Immun. 135, 3151 (1985)

\section{Genetic prediction of cystic fibrosis}

Sir-Brock and van Heyningen (Nature $319,184 ; 1986)$ have reason to be a little indignant about the way their current prenatal test for cystic fibrosis was ignored, but they are wrong to state that first trimester prenatal diagnosis by gene tracking with linked DNA probes suffers from a major problem due to the inability to confirm cystic fibrosis in the aborted embryo. Their "cardinal requirement of prenatal testing, namely that some method of confirmation of diagnosis on the abortus be available" only really applies to tests based on empirical correlations between the level of some factor in amniotic fluid, for example, and fetal genotype (tests such as Brock's current prenatal cystic fibrosis test and his earlier important contribution, the $\alpha$-fetoprotein test for open neural tube defects like spina bifida). This is not so for genetic prediction using linked probes.

The reliability of gene tracking using linked DNA probes depends on both exclusion of non-allelic genetic heterogeneity and the frequency of recombination between disease locus and marker(s). Careful linkage studies in a large number of families from different populations will allow exclusion of significant non-allelic heterogeneity and provide a mean recombination frequency. These studies are already well advanced. The recombination frequency combined with the prior genetic risk will allow a reliable prediction of the false positive and false negative rates for first trimester diagnosis; and the false negatives will also be observed after birth. Thus, the information required to assess the reliability of the prenatal test is available from studying living family members.

Our unit has probably had the greatest experience in Britain of using linked and gene specific probes for early prenatal diagnosis of haemophilia $\mathrm{A}$, a disorder for which there is a reliable second trimester test by fetal blood sampling. Our experience shows that couples at risk understand the inherent errors in using linked probes, and yet almost always opt for the earlier test based on chorionic villus sampling. often followed, in the case of a negative result, by fetal blood sampling. We cannot over-emphasize the advantages of early diagnosis as perceived by the family. These include the psychological benefits of an early result and an earlier and safer therapeutic abortion, if they choose that option. A consequence seems to be a shorter gap before trying again if unlucky. Furthermore, there is the opportunity to keep the pregnancy private until the matter is decided.

Marcus E. Pembrey Sue Malcolm Mothercare Unit of Paediatric Genetics, Institute of Child Health, University of London, 30 Guilford Street, London WC1N 1EH, UK

\section{Scientific Correspondence}

Scientific Correspondence is intended to provide a forum in which readers may raise points of a rather technical character which are not provoked by articles or letters previously published. 